Part of Journal of Research of the National Bureau of Standards, Volume 21, December 1938

\title{
METHOD FOR DETERMINING UNIFORMITY OF TEMPERATURE IN CRYOSTATS
}

\author{
By Martin Shepherd
}

\begin{abstract}
This note reports a sensitive method by which the uniformity of temperature in cryostats (or other constant-temperature baths) may be determined. The method involves the measurement of differences of saturation pressures of a suitable substance (which need not be critically pure) by means of a very easily constructed differential manometer. Such an instrument is capable of differentiating temperature effects at the surface and within the bath, and offers some interesting possibilities in studying low-temperature baths.
\end{abstract}

The platinum resistance thermometer and the differential thermel, which are normally employed to determine the uniformity of temperature within cryostats (or other constant-temperature baths) are subject to at least one annoying limitation. Neither instrument is well suited for measuring differences of temperature at the surface of the bath. While either instrument may be used in connection with a vapor-pressure thermometer to measure effects of temperature at the surface, the procedure required is relatively complicated and time-consuming.

It is possible to compare the temperatures at any two points within the bath or at its surface simultaneously, with extreme sensitivity and with little or no significant temperature lag, by means of a very simple differential saturation-pressure manometer. This manometer was previously described in connection with the determination of the purity of gases ${ }^{1}$ and is illustrated in figure 1 as modified for the present purpose.

Both sides are simultaneously filled with a suitable gas drawn from the same source. The gas used will determine the effective temperature range, which may be extended (with an increase in sensitivity) by filling to a pressure of several atmospheres. If an extended range of temperature must be studied, the manometer may be refilled with other gases, or a set of several permanent manometers can be made. Since the gas used need not be critically pure, and the manometer itself is easily constructed, the instrument possesses the practical advantages of relative cheapness and availability. If greater flexibility is desired, spiral expansion joints can be used to permit movement of the stems, or a flexible metal tubing may be substituted for

\footnotetext{
${ }^{1}$ BS J. Research 2, 1156 and 1169 (1929) RP75; 12, 185 (1934) RP643.
} 
the glass stems. The latter may be attached to the glass by any of the several methods available for making such joints.

A system of shields may be used in the various combinations shown, and the possibilities offered are attractive. For example, if both stems of the manometer are left bare (see 1 of the figure), the differential vapor pressures observed should indicate the combined effect of differences of temperature within the bath and at its surface. The

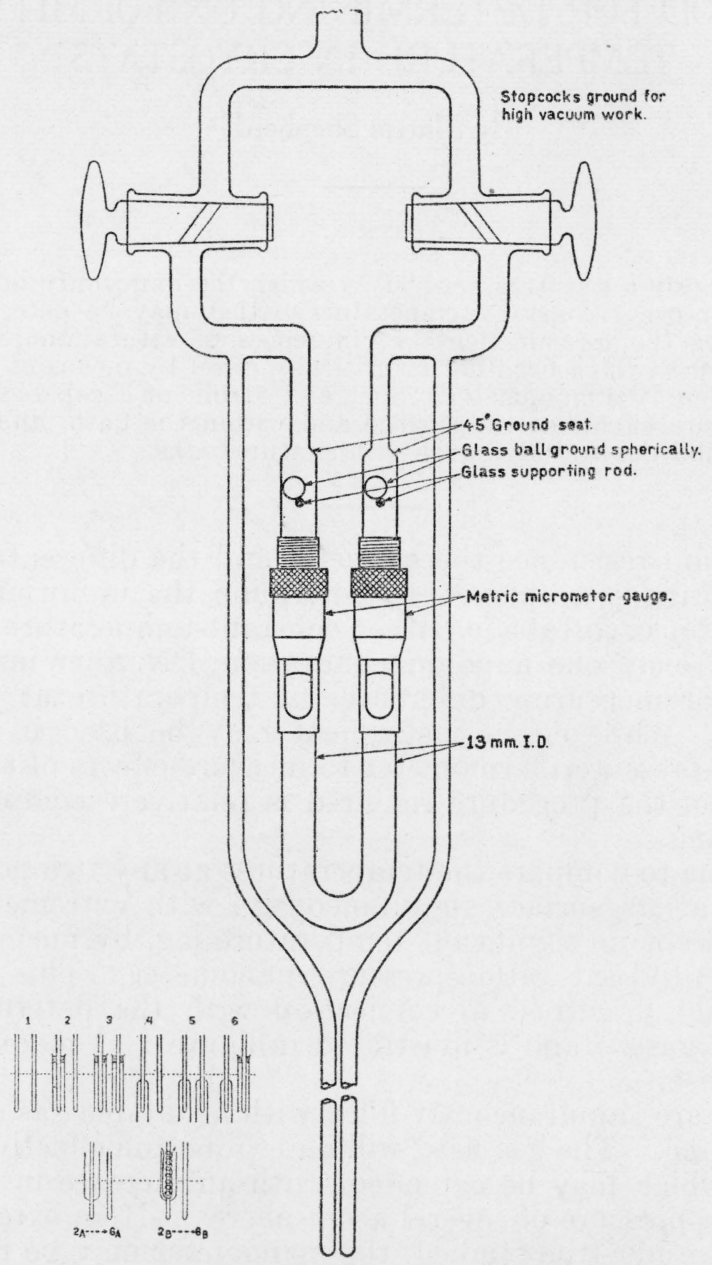

Figure 1.-Detail of differential manometer.

arrangement shown in $2,2 \mathrm{~A}$, or $2 \mathrm{~B}$ may be used to determine differences between a point within the bath and the effective temperature as measured by a simple vapor-pressure thermometer. This should immediately disclose the suitability of a bath intended for the comparison of temperatures indicated by a platinum resistance thermometer and a vapor-pressure thermometer. When both stems are shielded (as in 3), differences of temperature between two points 
within the bath may be determined. With one stem shielded as in 4, a difference between one spot at the surface and the effective temperature measured by a vapor-pressure thermometer may be indicated. Temperatures at two separate points on the surface may be simultaneously compared if the shields are arranged as in 5. The arrangement shown in 6 may be used to observe differences in temperature between one part of the surface and a point within the bath.

Thus the differential manometer might be used to study the uniformity of temperature more advantageously than is possible with either of the normally employed electrical instruments, since temperatures can be compared both within the bath and at the surface. Moreover, the difference of temperatures between two points is measured simultaneously and with high sensitivity. It is entirely possible to observe some erratic behavior of the bath, which might be missed if a resistance thermometer with a relatively large thermal lag were moved from point to point, by simply allowing the manometer to remain in a fixed position and watching the positions of the mercury menisci. By evacuating one side of the manometer and using it as a simple vapor-pressure thermometer, the constancy as well as the uniformity of temperature may be determined.

In studying the temperatures at the surface of a bath, the differential manometer possesses a unique advantage. It is always in the surface itself, even when the surface of the liquid is moving, uneven, and of constantly varying height. The immersed stem of the manometer responds quickly to changes of temperature at the surface. The fullest extent of temperature change will not be realized, since the thin glass stem interposes a heat capacity. But under similar conditions, the platinum resistance thermometer is obviously not suitable. The differential thermel, which does have a low heat capacity, nevertheless has the physical limitation that, if placed at the very surface, it will sometimes be immersed and sometimes not; while, if it is lowered so that constant immersion is achieved, the local effects of the surface may be substantially lost.

The importance of the effects of temperature at the surface of the bath may alone frequently justify the use of the manometer, even if it is employed as an auxiliary to the resistance thermometer or thermel. The extent to which surface temperatures may vary is perhaps not generally realized. The following experiment will illustrate what may sometimes be expected. During the preparation of pure oxygen to be used by this Bureau as a reference point on the International Temperature Scale, ${ }^{2}$ the purity of the oxygen was tested by the method described in the previous references (footnote 1). The bulbs of the differential manometer were thin-walled spheres touching one another, and were immersed in a bath of liquid oxygen boiling vigorously within a D'Arsonval tube. Oxygen of high purity was simultaneously condensed from a common source into each bulb. The saturation pressure within each bulb should have been identical, and the manometer should have indicated no difference in pressure, if the temperature of the bath had been uniform. Under these conditions, however, the manometer actually indicated rapidly changing pressures within the

\footnotetext{
2 This work was done early in 1933. The present note was originally prepared at the same time, but publication was delayed pending possible experimental work. The opportunity for this work has, unfor-
} tunately, never presented itself.

$107462-38-9$ 
bulbs, in such a manner that first one and then the other bulb exhibited the higher pressure. This dancing behavior was quite erratic; at times the pressures remained steady, while at other times variations of as much as $20 \mathrm{~mm}$ (of mercury) were apparent. (At the normal boiling point of oxygen, $10 \mathrm{~mm}$ corresponds approximately to $0.13^{\circ} \mathrm{C}$.) Stirring the bath of boiling oxygen did not greatly improve the condition. When the bulbs were surrounded with a block of frozen mercury, which extended around the stems and above the surface of the bath, the variations previously observed were effectively eliminated. The author has used such a device for many years in connection with other cryostat baths, and has always found it satisfactory. There has been no breakage of glass when the mercury is frozen around the condensing bulbs. Such an arrangement would seem a logical one to employ in case the temperatures at two points of a bath were required to be accurately the same, as, for example, when calibrating a platinum resistance thermometer against a vapor-pressure thermometer.

The oscillating behavior exhibited by the differential manometer in the case cited may have been caused by variations of temperature within the bath or at the surface. The saturation pressure indicated by a vapor-pressure thermometer will correspond closely to the lowest temperature to which it is exposed, which is usually at the surface of cold baths. Because of these facts, E. F. Mueller of this Bureau suggested the desirability of shielding the stem of this type of thermometer by means of glass envelopes such as are indicated in the figure. He further suggested the possibility of electrically heating the stem within the shield (see series $B$, fig. 1 ).

It is evident from the foregoing discussion that the differential manometer may be particularly useful in studying baths to be employed for vapor-pressure thermometers, since the effects of surface temperature are so important. It is also evident that the use of the instrument may develop some surprises that have not been indicated in this sort note. Its general applicability and suitability have not been demonstrated or experimentally compared with existing apparatus and methods. The lack of experimental study precludes definite statements, and in the argument between the cryostat and the manometer, it is not possible to predict the certain winner on an estimated score of points. It seems likely, however, that interesting facts concerning both may be revealed; and this seems particularly probable in the field of vapor-pressure thermometry.

Washington, August 26, 1938. 